\title{
Training Adults with Brain Injury How to Help-seek when Lost: A Pilot Study
}

\author{
Young Susan Cho and McKay Moore Sohlberg \\ Department of Special Education and Clinical Sciences, University of Oregon, Eugene, Oregon, USA
}

\begin{abstract}
There is no research on the assessment or treatment of help-seeking behaviours for individuals with traumatic brain injury (TBI). This paper describes the development of a protocol, NICE (Noticing you have a problem, Identifying the information you need for help, Compensatory strategies, Evaluating progress) to train helpseeking for adults with TBI when lost. Theoretical and treatment components from three empirically validated interventions that target social problem-solving and communication skills were adapted to develop NICE: the Group Interactive Structured Treatment for Social Competence (GIST), the Problem Solving Group Protocol (PSG) and Interpersonal Recall (IPR). Preliminary pilot data evaluating the efficacy are presented for three adult persons with TBI. All three participants improved on the Executive Function Route Finding Task (EFRT) and help-seeking behaviours when wayfinding. Help-seeking is a constitutive factor in the wayfinding process capable of improvement. Preliminary evidence supports further investigation of this group intervention.
\end{abstract}

Keywords: traumatic brain injury, help-seeking, problem solving, cognitive rehabilitation

\section{Introduction}

Effective help-seeking is a critical skill essential for independent community reintegration, safety and quality of life, particularly in vulnerable populations such as people with acquired brain injury (ABI) who encounter challenges in task completion due to cognitive impairments (Evans, 2009; Sloan \& Ponsford, 2013; Sohlberg, Fickas, Hung, $\&$ Fortier, 2007). Help-seeking relies on a composite of executive functions and social skills (Babbage et al., 2011; Rietdijk, Simpson, Togher, Power, \& Gillett, 2013) and is defined 'as any communication about a problem or troublesome event which is directed toward obtaining support, advice or assistance in times of distress' (Gourash, 1978, p. 414). People with cognitive impairments are susceptible to challenges in com- munity reintegration when failures in attention, memory, executive functions and problem solving interfere with the ability to carry out daily activities (Sohlberg \& Turkstra, 2011). The ability to recognise when to ask others for assistance and to communicate effectively the need for help is critical when a person's own problem solving and cognition are impaired. Despite this need, there is currently no research on either the assessment or treatment of help-seeking behaviours for persons with traumatic brain injury (TBI). Models of social communication and social problem-solving, however, may be useful for understanding help-seeking as a construct, and for developing help-seeking training protocols for persons with cognitive impairments, because they share many of the same requisite components (D’Zurilla, Nezu, \&

Address for correspondence: Young Susan Cho, Department of Special Education and Clinical Sciences, University of Oregon, 5284 University of Oregon Eugene, OR 97403,USA. E-mail: ysc@uoregon.edu 
Maydeu-Olivares, 2004; Wang, Chan, \& Shum, 2014). The present authors drew from this literature to develop a help-seeking training intervention, since effective help-seeking requires both social competence and social problem-solving skills. The authors used the functional domain of wayfinding to pilot the intervention. The purpose of this paper is to describe the development and initial piloting of a therapy protocol specifically targeting helpseeking for adults with brain injury.

\section{Social Problem-solving and Brain Injury}

There has been a paucity of clinical research examining help-seeking in the brain-injury population. A literature search conducted using the databases PsycINFO, Medline and MIT CogNet with a combination of help-seeking, help-seeking when lost, brain injury, traumatic brain injury, social cognition, social problem solving, and wayfinding as key words, revealed no articles describing helpseeking behaviour in persons with ABI. However, there have been several treatment studies evaluating interventions designed to improve a related construct, social problem-solving (Hawley \& Newman, 2010; Sherr, Langenbahn, Simon, Rath, \& Diller, 2003).

Social problem-solving encompasses the process of problem solving between individuals as it occurs in the natural environment or real world (Channon \& Crawford, 2010; Robertson \& Knight, 2008). A model of problem solving that has been revised over the years to incorporate a socially meaningful component is the Social Problem Solving Model (D'Zurilla et al., 2004). Although the model was originally developed to describe processes in healthy individuals, it holds promise for informing understanding of social problemsolving in the ABI population (Rath, Hradil, Litke, \& Diller, 2011; Rath, Simon, Langenbahn, Sherr, \& Diller, 2003; Sherr et al., 2003). Researchers identify three essential components of social problemsolving: (a) a learning process that can result in a change in social outcome; (b) general coping strategies relevant to a variety of problem environments; and (c) self-control methods for maintenance and generalisation (D'Zurilla \& Goldfried, 1971; D’Zurilla \& Nezu, 1982, 1990, 2007; D'Zurilla et al., 2004). The model also acknowledges that problem solving and emotion are directly related, and emotions may influence the efficiency of problem-solving performance (D'Zurilla \& Nezu, 2007; D'Zurilla et al., 2004). Problem-solving therapies that address the associated emotional substrates in addition to the cognitive issues may result in better treatment outcomes
(Bell \& D'Zurilla, 2009; Malouff, Thorsteinsson, $\&$ Schutte, 2007).

\section{Treatment of Social Problem-solving and Communication}

Social problem-solving training. Help-seeking relies on social problem-solving. One of the few social problem-solving treatment protocols for persons with brain injury that includes an emotional component is the Problem Solving Group (PSG) Protocol (Sherr et al., 2003). PSG is a manualised curriculum for delivering group treatment that focuses on training logical reasoning and strategies to facilitate emotional selfregulation for effective social problem-solving. Emotional self-regulation is conceptualised as a key self-initiated process to avoid costly errors during problem solving. It recognises that social problem-solving requires more than mere knowledge, it requires self-evaluation. In an experimental evaluation of the efficacy of PSG, researchers randomly assigned 60 outpatients with mild TBI to either a conventional group that focused on traditional neurological rehabilitation or a PSG group that focused specifically on treatment of problem-solving deficits. Results suggested that the PSG group treatment, focusing on the emotional aspects of social problem-solving, was more effective than a traditional cognitive remediation group treatment focusing only on improving basic problem-solving skills. Helpseeking performance can be affected by emotional constructs, such as self-confidence. In a wayfinding study, those participants with ABI who engaged in negative self-talk were less successful in their wayfinding, supporting the need for interventions that target self-confidence and self-efficacy (Cho \& Sohlberg, 2014).

Another therapy protocol that was designed to improve social communication by addressing social problem-solving in people with TBI is the Group Interactive Structured Treatment for Social Competence (GIST; Hawley \& Newman, 2010). The GIST programme consists of five phases: engagement, awareness, goal setting, skills mastery and generalisation. Development of the GIST protocol is based on established principles of cognitive behaviour therapy, group therapy and holistic neuro-rehabilitation. Similar to the PSG protocol (Sherr et al., 2003), the GIST uses a group therapy model that emphasises rehabilitation of cognitive, affective and behavioural impairments affecting social problem-solving. The GIST emphasises group process mediators: 'universality, the realization that one is not alone; altruism, the ability to be helpful to others; and group cohesion, a sense of 
belonging and group trust' (Hawley \& Newman, 2010, pp. 1293-1294). Social self-confidence is facilitated in the group environment to promote generalisation of treatment.

The GIST treatment was implemented using a randomised controlled trial and deferred treatment design with a volunteer sample of 52 persons with TBI who were at least 1 year post injury (Dahlberg et al., 2007). Most significantly, the treatment group improved on their general participation in conversation. Improvements were also noted in external relation, the listener's role in relating comments to the speaker's previous comments, and in internal relation, the speaker's role to ensure ideas are cohesive, relevant and related. Social style, clarity of expression and speech characteristics were also improved post treatment. The second hypothesis that overall satisfaction with life would be improved was supported at 3, 6 and 9 months' follow-up, but participant performance on the test measures did not significantly improve immediately after the treatment. Improvement in social communication skills was also supported by clinician ratings as well as participants' perceived skill ratings.

The efficacy of the GIST treatment protocol was also supported in a feasibility study to improve social communication skills in 30 persons with TBI with comorbid neurological or psychiatric aetiologies (Braden et al., 2010). Consistent with findings obtained by Dahlberg and colleagues (2007), improvements were noted in social communication skills. Together, these findings lend positive support for the improvement of social communication through targeted group intervention. Helpseeking requires effective social communication, as a person must interact with others to enlist assistance. The GIST programme thus provided foundational intervention components for the current help-seeking pilot intervention.

Social skills training. Social skills are a critical component of help-seeking (Bergstrom, Najdowski, \& Tarbox, 2012) as help-seeking requires social interactions with others when asking for assistance, generating questions and listening for responses (Cho \& Sohlberg, 2014). Several researchers have evaluated the efficacy of directly training discrete social skills in people with $\mathrm{ABI}$ (Bornhofen \& McDonald, 2008; Dahlberg et al., 2007; McDonald et al., 2008; Ylvisaker, 2006). Snow (2013) states 'communication, by its nature, is context bound' (p. 153) suggesting that lack of generalisation of training discrete social skills is due to training in decontextualised environments that do not approximate real-life encounters. More recent research emphasises the need for 'learning meaningful skills in meaningful contexts' because even under optimal conditions, generalisation of treatment skills is poor (Snow, 2013; Ylvisaker, 2003). These researchers integrate direct instruction of social skills using modelling and guided practice (Engelmann \& Carnine, 1982; Horner, Sprague, \& Wilcox, 1982) with cognitivebehavioural techniques that help individuals selfreflect in order to understand the thoughts and feelings that influence their behaviour (Heish et al., 2013; Silverberg et al., 2013). Interpersonal Recall (IPR) (Helffenstein \& Wechsler, 1982) is an example of a therapeutic approach that incorporates both direct instruction and self-reflection for the purpose of improving individual social skills in people with brain injury (Helffenstein \& Wechsler, 1982). Participants review videotaped social interactions of themselves with a helper and receive coaching. The goal of the review is not to teach a new skill, but rather, to explore thoughts and feelings that will lead to identification of behaviours characterising the desired social interaction. This is supplemented by opportunities to practise the identified behaviour. IPR was used successfully in a controlled trial to target social skills deficits in persons with brain injury (Helffenstein \& Wechsler, 1982).

In summary, studies to date provide several models of efficacious training methods for improving social communication (Braden et al., 2010; Dahlberg et al., 2007; Hawley \& Newman, 2010) and social problem solving (Rath et al., 2003; Sherr et al., 2003). The authors drew from this literature to develop a help-seeking training protocol and used wayfinding as an instructional domain to pilot the intervention. Both the PSG and GIST were used as models to develop a protocol specific to training help-seeking in people with brain injury called NICE (Noticing you have a problem, Identifying the information you need for help, Compensatory strategies, $\boldsymbol{E}$ valuating progress). The NICE protocol incorporates key aspects of social problem solving that have been shown to be critical and amenable to rehabilitation. Similar to the PSG and GIST, NICE instructs social problem-solving using modelling, reflection, reinforcement and feedback (Cho \& Sohlberg, 2014). Unlike previous studies, the NICE protocol was developed to train help-seeking within a particular activity domain, wayfinding.

\section{Wayfinding: A Domain for Studying Help-seeking}

Wayfinding is an activity of daily living that is frequently disrupted following $\mathrm{ABI}$ and has been identified as critical for community reintegration, 
safety and quality of life (Antonakos, 2007; Lui et al., 2008; Skeleton, Ross, Nerad, \& Livingstone, 2006; Sohlberg et al., 2007). Navigation profiles developed for people with brain injury revealed that fear of getting lost prevented people from venturing into the community (Lemoncello, Sohlberg, Fickas, \& Prideaux, 2010). Wayfinding requires problem-solving and help-seeking skills, abilities that are commonly impaired following brain injury (Sohlberg et al., 2007; van Asselen et al., 2005).

In spite of its importance, studies of wayfinding after brain injury have narrowly focused on route finding and have ignored issues of trouble shooting and help-seeking (Skeleton et al., 2006). One exception was a comparison of wayfinding performance of 18 adults with TBI to matched controls (Lemoncello et al., 2010). Participants followed written directions along an eight-step route in an unfamiliar neighbourhood with three intentionally challenging areas where choices needed to be made. They were encouraged to use a cellular phone to request assistance if they became lost. Participants with TBI demonstrated significantly greater route wayfinding errors and hesitancy than the controls. They requested assistance over the phone more frequently but had trouble using the help. This study highlights the difficulty with wayfinding and help-seeking behaviour in the TBI population.

No studies have examined naturalistic, selfinitiated help-seeking behaviours of adults with brain injury when lost, nor are there evaluations of interventions to improve help-seeking. However, researchers have examined help-seeking behaviours of children with autism when lost, who, similar to adults with brain injury, exhibit impairments in social communication and problem solving (Bergstrom et al., 2012). Bergstrom and colleagues (2012) trained three children with autism to ask for help from store employees when they were separated from their caregivers. Help-seeking behaviours improved and generalised for all participants after implementation of a treatment package.

Behavioural interventions with a goal to indirectly improve wayfinding by treating impairments in awareness of space have been largely unsuccessful (Butter \& Kirsch, 1992, 1995). Successful wayfinding requires the use of effective compensatory strategies (Carlson, Holscher, Shipley, \& Dalton, 2010; Spiers \& Maguire, 2008) as well as help-seeking. Even when provided with compensatory strategies, naturalistic demands and contextual variability will result in a need for troubleshooting as the real world does not stay static. In real-world environments, good navigators use different and more flexible compensatory strategies (Kato \& Takeuchi, 2003; Saucier et al., 2002) and poor navigators use strategies relying on verbal sequential lists of directions (MacFadden, Elias, \& Saucier, 2003). The NICE protocol (Cho \& Sohlberg, 2014) piloted in this study was developed to address this need to train adults with brain injury how to ask effectively for help with directions when lost, using compensatory strategies.

\section{Nice: A Help-Seeking Training Protocol}

\section{Development of NICE}

The NICE protocol (Noticing you have a problem, Identifying the information you need for help, Compensatory strategies, Evaluating progress) is a curriculum-based group intervention protocol targeting help-seeking behaviours during wayfinding. NICE was developed using components of empirically validated interventions that target social problem-solving and social communication skills, including the GIST (Hawley \& Newman, 2010), PSG (Sherr et al., 2003) and IPR (Helffenstein \& Wechsler, 1982). These protocols were selected as they had all been evaluated using controlled trials and they addressed social problem-solving skills important for help-seeking. Permission was granted by Principal Investigators of the GIST and PSG to modify and use the protocols for development of NICE. Below is a detailed summary of the NICE protocol to provide researchers with sufficient description to encourage future development and evaluation. The NICE protocol is provided as supplementary Appendix A, available online.

\section{NICE Training Protocol}

The first step of NICE, Noticing you have a problem, requires participants to log experiences specific to problems encountered during wayfinding. Participants are trained to log what, why and where a breakdown occurs to encourage problem recognition. The second step of NICE, Identifying the information you need for help, focuses on teaching direct help-seeking behaviours such as paying attention to the speaker, expressing the right idea, being polite, maintaining appropriate space and having social confidence. The third step of NICE, Compensatory strategies, focuses on developing a personal strategy list to use during the problem-solving process. The last step of NICE, Evaluate progress, focuses on training participants to analyse their help-seeking behaviour and generate plans for initiating future social problemsolving when lost. The last step of NICE encourages self-evaluation and self-correction when necessary. 
NICE session structure. Each of the six sessions follows roughly the same group structure modelled in the GIST programme, where each session begins with identifying the purpose of the session, followed by review of previous concepts, introduction to a new topic, role-play practice and homework assignment (see supplementary Appendix A). The importance of working in a group environment to facilitate learning, support and feedback is discussed in the first session. The purpose of using the group format to implement NICE is to teach persons with brain injury how to recognise when they are lost, generate a solution and ask for help in a manner that is appropriate, effective and efficient. Group treatment provides opportunities for members to learn from each other's successes and failures, and facilitates feelings of universality that may increase social confidence and motivation (Hawley \& Newman, 2010).

Video-taped interactions are used to teach and reinforce social communication skills (Hawley \& Newman, 2010; Helffenstein \& Wechsler, 1982). Video feedback is a powerful tool for improving communication skills and allows patients to observe the present, analyse the past and plan for the future (Rath et al., 2003). In a recent randomised control trial (RCT), video feedback was associated with significantly fewer errors during the execution of functional tasks by participants with brain injury compared to alternative feedback strategies (e.g., experiential feedback, verbal feedback). Results suggested that video feedback was a powerful metacognitive treatment strategy that facilitated independent self-regulatory behaviour (Schmidt, Fleming, Ownsworth, \& Lannin, 2012). In the NICE protocol, video feedback allows group members to practise new skills in a safe environment. Multiple viewpoints and suggestions are discussed to improve cognitive flexibility and generate alternative solutions and behaviours.

All NICE sessions include practice role plays of target help-seeking skills. Role play can be an effective treatment technique to teach specific social skills (Rath et al., 2003). Role plays allow a patient to actually 'do' rather than 'talk' about real-life social scenarios. NICE sessions include practice role plays during the group treatment time as well as opportunities for individual video-taped role plays with unfamiliar confederates immediately after the session, to promote generalisation of skills. Consistent with the PSG (Rath et al., 2003), the purpose of the NICE role play is to help participants learn, master and generalise concepts to new contexts. One modification made with the NICE protocol was to combine IPR (Helffenstein \& Wechsler, 1982) with the individual role play scenarios in Sessions 5 and 6 to provide participants with opportunities to learn how to provide and accept feedback from group members (Table 1).

\section{Method}

The NICE protocol was piloted with a convenience sample of three participants. The implementation and results of the pilot are described in this section to examine the primary research hypothesis that the NICE intervention targeting help-seeking behaviour would improve both navigation for adults with brain injury and independent help-seeking behaviours during wayfinding.

\section{Participants}

Participants were recruited from a local assisted living facility for people with acquired brain injury. Facility staff were presented with the goals of the study and the criteria for participation and asked to refer residents they thought would benefit from assistance with help-seeking when wayfinding. Inclusion criteria included: (a) sufficient English fluency/literacy; (b) documented brain injury by a medical doctor; (c) stated desire to improve navigation skills; (d) stated motivation to participate; (e) adequate hearing and visual acuity; and (f) access to transportation to the research clinic. Exclusion criteria included the presence of: (a) psychiatric disorder(s); (b) active substance abuse; (c) motor limitations, sensory or other neurological limitations affecting the ability to participate in the training or wayfinding; and (d) language impairments preventing comprehension of the NICE protocol. All participants were consented in accordance to the university review board policies. Four participants met the criteria, and three participants completed the NICE treatment. One individual discontinued treatment due to substance abuse issues. Participants were paid US\$50 upon completion of the project.

Participant S.F. Participant S.F. was a 51-yearold male 3 years post onset of TBI secondary to a physical altercation with several unknown persons. No loss of consciousness was noted in the medical report at the time of injury. He completed 14 years of education and, prior to injury, was employed as an internet programmer. S.F. self-reported difficulty when needing to ask for help from strangers and following verbal directions to a goal destination. Inappropriate use of humour and language was noted at the time of initial interview, as well as limited eye contact with the examiner. Chronic symptoms reported by a case worker included mild cognitive impairments in attention and short-term memory. The case worker stated that S.F. often locked himself out of the apartment and missed 
TABLE 1

Development of NICE

\begin{tabular}{|c|c|c|c|}
\hline Session & NICE & Session Content & Research Based Model \\
\hline Session 1: Orientation & $\begin{array}{l}\text { Give overview of NICE } \\
\text { programme and } \\
\text { introduce group } \\
\text { participants } \\
\text { Teach step } 1 \text { of NICE - } \\
\text { Noticing you have a } \\
\text { problem }\end{array}$ & $\begin{array}{l}\text { Purpose of the group } \\
\text { What is communication } \\
\text { and why do we need } \\
\text { good communication } \\
\text { for help-seeking when } \\
\text { lost? } \\
\text { Behaviours necessary } \\
\text { for good } \\
\text { communication }\end{array}$ & $\begin{array}{l}\text { Adapted from the PSG: } \\
\text { Clear Thinking } \\
\text { worksheets }\end{array}$ \\
\hline $\begin{array}{l}\text { Sessions } 2 \text { and 3: Skills } \\
\text { of a Great } \\
\text { Communicator }\end{array}$ & $\begin{array}{l}\text { Practise how to ask for } \\
\text { help } \\
\text { Teach step } 2 \text { of NICE - } \\
\text { Identifying the } \\
\text { information you need } \\
\text { for help }\end{array}$ & $\begin{array}{l}\text { Characteristics of a } \\
\text { great communicator } \\
\text { Why is communication } \\
\text { important when you } \\
\text { are lost and need to } \\
\text { ask for help? } \\
\text { How to be a great } \\
\text { communicator when } \\
\text { asking for help }\end{array}$ & $\begin{array}{l}\text { Adapted from the GIST: } \\
\text { Skills of a Great } \\
\text { Communicator }\end{array}$ \\
\hline $\begin{array}{l}\text { Session 4: Developing } \\
\text { Social Confidence } \\
\text { with Positive Self-talk }\end{array}$ & $\begin{array}{l}\text { Identify confidence and } \\
\text { self-talk } \\
\text { Teach step } 3 \text { of NICE - } \\
\text { Compensatory } \\
\text { strategies }\end{array}$ & $\begin{array}{l}\text { Discuss social } \\
\text { confidence and } \\
\text { feelings when lost } \\
\text { Identify how to use } \\
\text { positive self-talk and } \\
\text { change negative } \\
\text { self-talk }\end{array}$ & $\begin{array}{l}\text { Adapted from the PSG: } \\
\text { Personal Strategy List } \\
\text { Adapted from the GIST: } \\
\text { Developing Social } \\
\text { Confidence with } \\
\text { Positive Self-Talk }\end{array}$ \\
\hline $\begin{array}{l}\text { Sessions } 5 \text { and 6: } \\
\text { Video-taping and } \\
\text { Evaluation }\end{array}$ & $\begin{array}{l}\text { Learn to give and } \\
\text { accept feedback } \\
\text { Teach step } 4 \text { of NICE - } \\
\text { Evaluating progress }\end{array}$ & $\begin{array}{l}\text { Watch videos with the } \\
\text { group } \\
\text { Discuss rationale and } \\
\text { rules for giving video } \\
\text { feedback for practice } \\
\text { role-played scenarios } \\
\text { Share and accept } \\
\text { feedback from others }\end{array}$ & $\begin{array}{l}\text { Adapted from the PSG: } \\
\text { Observing behavior } \\
\text { in the present and } \\
\text { analyzing past } \\
\text { behavior for future } \\
\text { planning } \\
\text { Adapted from the GIST: } \\
\text { Video-Taping and } \\
\text { Social Problem } \\
\text { Solving } \\
\text { Adapted modified } \\
\text { version of IPR: Group } \\
\text { Video feedback }\end{array}$ \\
\hline
\end{tabular}

PSG, Problem Solving Group Protocol; GIST, Group Interactive Structured Treatment for Social Competence; IPR, Interpersonal Recall.

scheduled appointments. S.F. stated he wanted to participate in the NICE training to gain independence while wayfinding in the community.

Pre-treatment performance on the Executive Function Route Finding Task (EFRT; Cho, Sohlberg, \& Boyd, 2012) was characterised by point deductions in task understanding, information seeking, retaining directions, error detection and error correction for both routes (see Figure 1A). Also, S.F. distorted peripheral details while recalling the goal destination, requiring clarification and/or cues to self-monitor by the examiner. A difference in pre-treatment performance was noted during the two different routes, suggesting a possible practice effect, which across repeated administrations are a common finding (Sohlberg, Boyd \& Cho, 2012). Social behaviour while help-seeking was also rated during the EFRT routes when participants requested assistance from helpers during the wayfinding task. Points were deducted on the social behaviour rating scale when inappropriate behaviours were observed when getting the attention of the helper (e.g., 'Hi sweetie pie.') and 


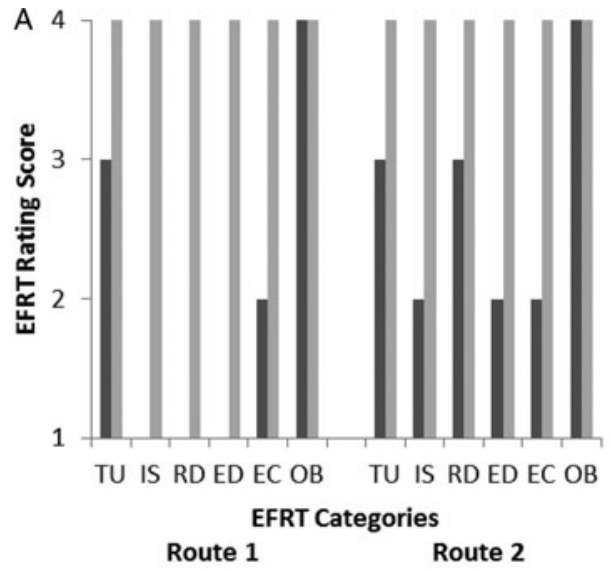

- Pre-Treatment (Route 1= Fronhnmayer Music School; Route 2= Lillis Business Complex)

nost-Treatment (Route 1= HEDCO Education; Route 2= Knight Library)

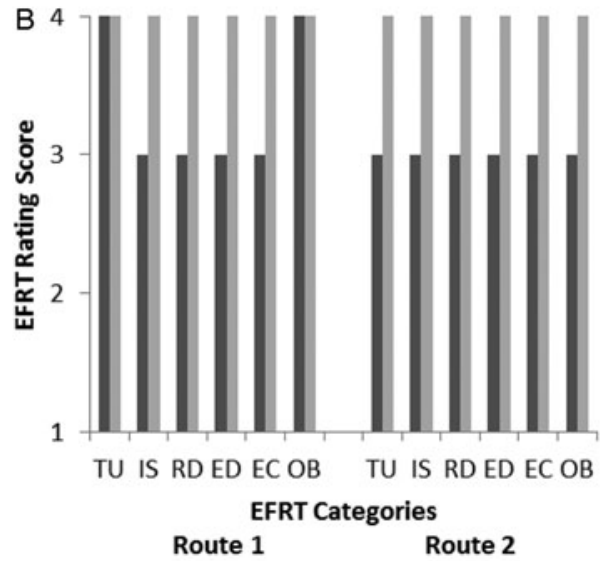

- Pre-Treatment (Route 1= Knight Library; Route 2= Fronhnmayer Music School)

- Post-Treatment (Route 1= Lillis Business Complex; Route 2= HEDCO Education)

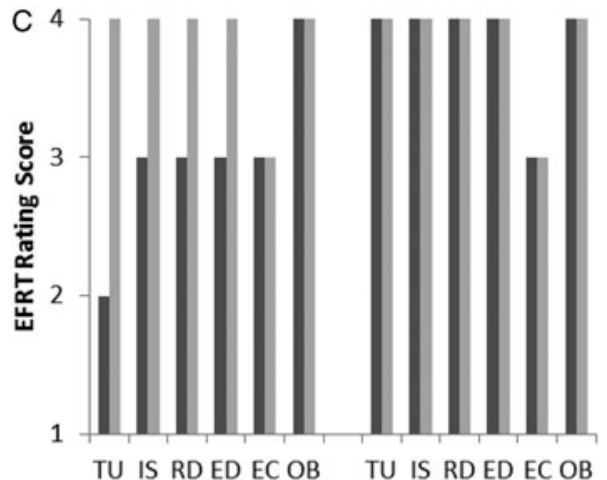

- Pre-Treatment (Route 1= Fronhnmayer Music School; Route 2= HEDCO Education)

- Post-Treatment (Route 1= Knight Library; Route 2= Lillis Business Complex)

\section{EFRT Categories}

Route 1

Route 2

\section{FIGURE 1}

Pre- and post-treatment EFRT rating scores for Route 1 and Route 2 for each category: (A) participant S.F.; (B) participant M.A.; (C) participant G.B. TU = Task understanding; $I S=$ information seeking; $R D=$ retaining directions; $E D=$ error detection; $\mathrm{EC}=$ error correction; $\mathrm{OB}=$ on-task behaviour. 


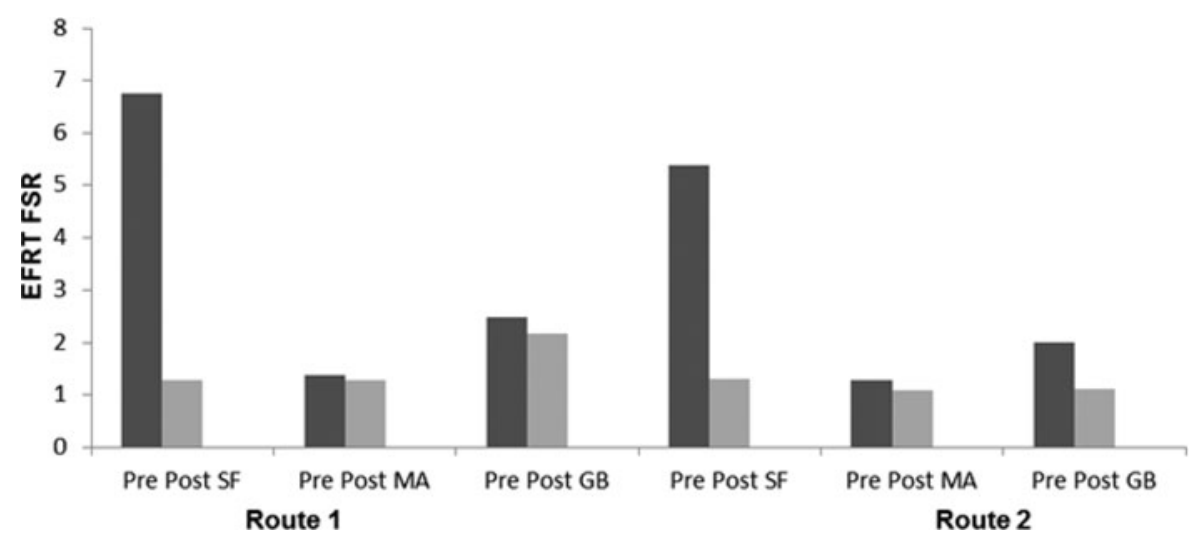

FIGURE 2

Footstep ratio scores for the three participants (SF, MA and GB) pre- and post-treatment for Routes 1 and 2.

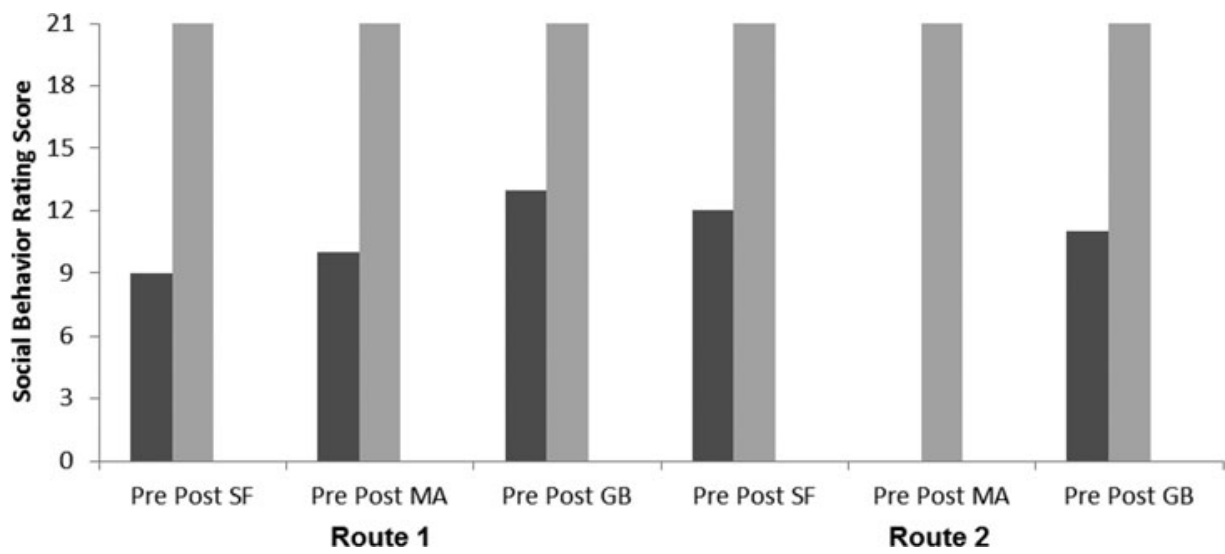

FIGURE 3

Social behaviour rating scores for the three participants (SF, MA and GB) pre- and post-treatment for Routes 1 and 2.

negative self-talk was apparent (e.g., 'I am crazy, stupid, dumb.'). S.F. often walked away from the helper before getting the full directions and no eye contact was observed with any of the helpers during the help-seeking attempts (see Figure 3).

Participant M.A. Participant M.A. was a 24-yearold male 4 years post onset of TBI secondary to a longboarding accident without a helmet. Medical reports indicate loss of consciousness for 3 days after the injury with an initial Glasgow Coma Scale (GCS) of 3. Post-traumatic amnesia (PTA) duration was not documented. He completed 12 years of education and, prior to injury, was employed in retail. M.A. self-reported difficulty navigating to non-routine routes unassisted. At the time of interview limited eye contact with the examiner was noted, and M.A. asked questions without giving the examiner adequate time to respond before changing the topic. Chronic symptoms reported by his case worker included mild cognitive impairment in short-term memory, impulsivity and poor conversational turn-taking. M.A. stated that he wanted to participate in the NICE training to navigate independently without assistance in the community.

Pre-treatment performance on the EFRT (Boyd $\&$ Sautter, 1993) was characterised by point deductions in information seeking, retaining directions, 
error detection and error correction for Route 1 and for task understanding, information seeking, retaining directions, error detection, error correction and on-task behaviour for Route 2 (see Figure 1B). Social behaviour while help-seeking was also rated during the EFRT routes when participants requested assistance from helpers during the wayfinding task but only for Route 1 . Helpseeking was not self-initiated on Route 2 because a passerby offered assistance before M.A. could request help. For the one pre-treatment EFRT route, points were deducted on the social behaviour rating scale when M.A. displayed difficulty paying attention to the helper (e.g., maintaining eye contact) and expressing the right idea (e.g., asking the navigation/destination question clearly and concisely). No compensatory strategies were used to remember information (see Figure 3).

Participant G.B. Participant G.B. was a 52-yearold male 26 years post onset of TBI secondary to explosion of a self-made bomb. Medical reports indicate loss of consciousness for 11 days after injury with no GCS or PTA duration documented. He completed 12 years of education and, prior to injury, was employed as an auto technician. G.B. self-reported that he seldom navigated to unfamiliar places unassisted by a caregiver. At the time of interview, no eye contact was attempted with the examiner. Chronic symptoms reported by his case worker included lack of assertiveness and judgement when decision making (e.g., would defer all decision making to caregiver) and poor inferencing skills. G.B. stated that he wanted to participate in the NICE training to navigate independently to unfamiliar places without his caregiver.

Pre-treatment performance on the EFRT (Boyd \& Sautter, 1993) was characterised by point deductions in task understanding, information seeking, retaining directions, error detection and error correction for Route 1 and point deductions in error correction for Route 2 (see Figure 1C). A difference in pre-treatment performance was noted during the two different routes, suggesting a possible practice effect. Social behaviour was also rated during the EFRT administration when participants requested assistance from helpers during the wayfinding task. Points were deducted on the social behaviour rating scale when G.B. displayed difficulty getting attention of the helper, paying attention the helper, and clearly expressing his needs. No compensatory strategies were used to remember information and negative self-talk was noted throughout the task (see Figure 3).

\section{Measurement of Help-seeking during Wayfinding}

There are no existing measurements of helpseeking in the context of wayfinding. We developed two measures to evaluate the efficacy of the NICE protocol. The first was an extension of an existing route-finding measure, the Executive Function Route-Finding Task (EFRT; Boyd \& Sautter, 1993). The EFRT is an ecological measure of wayfinding behaviour originally designed to evaluate the integrity of executive functions following brain injury (Boyd \& Sautter, 1993; Chaytor \& Schmitter-Edgecombe, 2003; Manchester, Priestley, \& Jackson, 2004). The examiner observes the participant navigating to an unfamiliar destination and rates performance on a four-point scale to measure the degree to which the participant is dependent on the examiner for the following domains: (a) task understanding; (b) information seeking; (c) retaining directions; (d) error detection; (e) error correction; and (f) on-task behaviour. This results in the EFRT Total Score. Higher scores on the EFRT indicate more independent wayfinding. Wayfinding behaviours and use of strategies are also documented in a narrative. The EFRT has been found to differentiate between a clinical population and healthy controls, with good construct validity (Kizony, Demayo-Dayan, Sinoff, \& Josman, 2011; Spikman, Deelman, \& van Zomeren, 2000; Webber \& Charlton, 2001) and has high inter-rater reliability (Boyd \& Sautter, 1993, Kizony et al., 2011; Spikman et al., 2000).

An earlier study increased the objectivity of the EFRT by adding a footstep ratio (FSR) to measure efficiency of wayfinding (Cho et al., 2012). FSR is calculated by dividing the participant's actual number of footsteps by the examiner's fewest number of footsteps, using a pedometer on routes that have been calibrated. The use of the EFRT allowed us to determine the potential impact of the NICE protocol on wayfinding performance. A lower footstep ratio indicates that fewer footsteps were taken to the goal destination, suggesting greater efficiency in wayfinding. The EFRT was administered and scored by the first author.

A second measure of help-seeking using structured role plays was developed with the goal of providing a repeated measure that would directly evaluate generalisation of the NICE components in social interaction. Role plays were administered by two trained confederates and scored by both the first author and graduate student clinicians to establish reliability. Five role-play scenarios with live confederate helpers available to assist the participant in attempting to find a destination were developed: (a) the helper speaks too 
fast without adequate time to remember information; (b) the helper is rude; (c) the helper gives poor directions; (d) the helper shares information, but clearly states that she did not know the goal destination; and (e) the helper distracts the participant with irrelevant information/questions. A four-point social behaviour rating scale (total 21 points) was used to rate seven performance parameters during the role plays, which were directly taught as part of the NICE protocol: (a) getting attention of the helper (e.g., excuse me); (b) paying attention to the helper (e.g., maintaining eye contact with the helper); (c) expressing idea (e.g., asking the navigation/destination question concisely); (d) using compensatory strategies to remember information relayed by the helper (e.g., requesting for clarification); (e) maintaining appropriate space (e.g., respecting personal space with the helper); (f) having social confidence (e.g., using affirmation statement and/or positive self-talk); and (g) being polite (e.g., tone). Points were received on each of the seven performance parameters when strategies were implemented (e.g., no strategy used $=0$; strategy use attempted but not successful $=1$; strategy use attempted and somewhat successful $=2$; strategy use attempted and goal achieved $=3$ ).

The role-play measures were piloted to establish reliability and validity. Prior to initiating the study, inter-rater reliability was established by two speech-language pathologists who rated live role plays acted out by two graduate student clinicians using the same five scenarios as those used with participants in the project (supplementary Appendix B). Interobserver agreement (IOA) was calculated for $100 \%$ of all sessions for each participant by [(Frequency of observations with agreement/ total number of observations $) \times 100 \%$ ] and was found to be $94 \%$. It should be noted that many standardised measures identified as ecologically oriented instruments fall short of this claim, because only a subset have been validated empirically to accurately capture skills necessary for everyday cognition (Rabin, Burton, \& Barr, 2007). The approximation of reallife tasks is a foundation of ecological validity but traditional standardised executive measures often lack ecological validity (Kizony et al., 2011; Spikman et al., 2000). We attempted to address the need to use an ecologically valid measure for the role plays by using scenarios from real-life helpseeking encounters experienced by participants in a prior investigation (Cho et al., 2012).

\section{Procedures}

The EFRT was administered at the University of Oregon campus using two pre- and two post- treatment routes. All routes were equated for distance (e.g., 294 feet), number of turns (e.g., 5) and floor level change (e.g., two floors $=1$ floor level change). There was a slight variation in footsteps (e.g., 130-140 steps) dependent on building stairs. A second rater who was not involved in the study verified the final measurements (e.g., number of steps) and an average was taken across four trials. Two locations started on a top level and ended downstairs; two locations started on a bottom level and ended upstairs. Each participant selected route order randomly by picking numbers out of a bag. The equivalence and validity of these routes was established in an earlier study (Cho et al., 2012). The EFRT was administered individually to all participants by the first author within 2 weeks before beginning the NICE group intervention. It was administered again by the first author within 1 week of completing the NICE intervention.

The first author conducted the treatment groups once weekly for 6 weeks at a university clinic. Each group treatment session lasted $60 \mathrm{~min}$ and followed the aforementioned structure. The three participants who completed the intervention attended all six sessions. Prior to each session, participants completed a role-play probe using the scripted role play with two trained confederates. Participants were individually escorted to a separate room to complete the video-taped role play. As described, each problem scenario presented an element of conflict and the participant had a choice of two confederates available to request for help when lost (see supplementary Appendix B). The confederates were trained by the first author during five 30-min sessions in order to ensure consistent application of the script. Performances on the role plays were scored independently by two speechlanguage pathologists who were not involved in the treatment, and ratings compared by calculating inter-rater agreement, using percent agreement on items, were found to be $91.5 \%$.

\section{Results}

Following completion of the NICE intervention, all three participants improved on the EFRT (Cho et al., 2012), both in terms of a reduced footstep ratio and on the EFRT Total Score. Participants showed variable improvements on the role play. Results supported the hypothesis that group intervention on help-seeking would positively impact wayfinding.

Participant S.F. Post-treatment performance data revealed improvement on the EFRT Total Score, with specific improvement in ratings of task 
understanding, information seeking, retaining directions, error detection and error correction (Figure 1A). Additionally, S.F. exhibited a reduction in footstep ratio (FSR) indicating he was taking fewer steps to reach the goal destination (Figure 2). No examiner cues were required for task completion during the post-treatment assessment. On the role play, S.F.'s social behaviour rating score improved from 9 to 21 points on Route 1 and 12 points to 21 points on Route 2 . Specific improvements were noted in the following areas: getting attention of the helper, reducing negative self-talk and waiting for a response from the helper when asking for help during the EFRT wayfinding task (Figure 3).

Participant M.A. Post-treatment performance data revealed improvement on the EFRT Total Score, with specific improvement in ratings of information seeking, retaining directions, error detection and error correction (Figure 1B). A reduction in FSR was noted from pre- to post treatment, indicating that M.A. was taking fewer steps to reach to the goal destination (Figure 2). On the role play, M.A.'s social behaviour rating score improved from 11 to 21 points on Route 1. Helpseeking was not self-initiated on pre-treatment Route 2 because a passerby offered assistance before participant M.A. could request help. Specific improvements were noted in the following areas: getting attention of the helper, paying attention to the helper, expressing the right idea, reducing negative self-talk, using compensatory strategies and waiting for a response from the helper (Figure 3). M.A. also self-reported that he felt more confident taking on the role of a helper post NICE treatment and giving directions to others, not just asking for directions from others.

Participant G.B. Post-treatment performance data revealed improvements on the EFRT Total Score, with specific improvement in ratings of all areas, with the exception of error correction for Route 1 (Figure 1C). A reduction in FSR was noted from pre- to post treatment, indicating that G.B. was taking fewer steps to reach to the goal destination (Figure 2). On the role play, G.B.'s social behaviour rating score improved from 13 to 21 points on Route 1 and 11 to 21 points on Route 2 . Specific improvements were noted in the following areas: getting attention of the helper, waiting for a response from the helper and reducing negative self-talk (Figure 3). G.B. and his caseworker also reported more instances of positive self-talk outside of the treatment sessions.

\section{Discussion}

All three participants exhibited diverse cognitive and social profiles and successfully completed the 6-week NICE treatment. Post-treatment measures suggested they were more efficient in their wayfinding after being taught help-seeking skills. Specifically, there was a reduction in their EFRT footstep ratio. In addition, improvements on the EFRT Total Score suggested they were more effective in their social interactions. Specific to helpseeking behaviour, each of the three participants demonstrated improvements during the in-session role plays. Improvement in role play was shown by score gains on the social behaviour rating scale specifically developed for use in this study. These preliminary results support the further development and evaluation of the NICE protocol to train help-seeking.

\section{Study Limitations}

The pilot investigation was exploratory, thus it had a number of limitations and results should be interpreted with caution. Participant information was available from files at the facility, but there was no verification of diagnoses or injury severity. The small $N$, homogeneous setting and restricted participant information limit the generalizability of the findings. Furthermore, the first author was both the primary developer and the lead researcher, thus subject to bias.

There were also limitations in the instruments used to measure treatment efficacy. Although the clinical utility of the EFRT has been improved recently by increasing the reliability of a quantitative index and providing normative performance data, there is no help-seeking score within the EFRT test itself. Therefore, the 21-point social behaviour rating scale was developed exclusively for use with the NICE protocol to measure help-seeking behaviours directly during navigation. Similarly, the role plays and scoring system were developed and piloted for this study and thus are limited in their validity. That said, it is hoped these two measurements will provide a foundation for larger validation, as there is a need for ecological measures of wayfinding and help-seeking. It is hoped that this exploratory, descriptive study provides the first step in preparing for experimental investigation.

\section{Conclusion}

Help-seeking behaviour is essential for solving problems when wayfinding. Although the NICE protocol was implemented with a small sample of participants with TBI in a descriptive study, these preliminary results support the hypothesis that 
intervention targeting help-seeking behaviour may improve navigation for adults with brain injury, and encourage further development and evaluation of the NICE protocol. Adults with TBI will most likely need to seek help on occasions when they do not have the internal resources to solve problems while navigating/route finding in the community. This project used the domain of wayfinding to train this important life skill, with emphasis on requesting assistance when lost. Wayfinding is a necessary skill; however, most people with cognitive impairment cannot do it alone, requiring assistance of others, which may not always be possible if they live alone. The NICE protocol, adapting theoretical and treatment components from the GIST (Hawley \& Newman, 2010), the PSG (Sherr et al., 2003) and IPR (Helffenstein \& Wechsler, 1982) is the first intervention designed specifically to teach people with TBI the necessary help-seeking behaviours to use when lost.

\section{Supplementary Material}

To view supplementary material for this article, please visit http://dx.doi.org/10.1017/BrImp. 2015.4.

\section{Acknowledgements}

This investigation was funded by the Evelyn $\mathrm{H}$. Bullock Fund. We gratefully acknowledge Dr Leonard Diller, Ms Jody Newman and Ms Lenore Hawley for their thoughtful comments on earlier versions of this manuscript. Portions of this article were presented in preliminary form at the 2013 American-Speech-Language-Hearing Association Convention and 2014 International Neuropsychological Society conference.

\section{References}

Antonakos, C.L. (2007). Compensatory wayfinding behavior in topographic disorientation from brain injury. Journal of Environmental Psychology, 24, 495502 .

Babbage, D.R., Yim, J., Zupan, B., Neumann, D., Tomita, M.R., \& Willer, B. (2011). Meta-analysis of facial affect recognition difficulties after traumatic brain injury. Neuropsychology, 25(3), 277-285.

Bell, A.C., \& D'Zurilla, T.J. (2009). The influence of social problem solving ability on the relationship between daily stress and adjustment. Cognitive Therapy and Research, 33, 439-448.

Bergstrom, R., Najdowski, A.C., \& Tarbox, J. (2012). Teaching children with autism to seek help when lost in public. Journal of Applied Behavior Analysis, 45(1), 191-195.
Bornhofen, C., \& McDonald, S. (2008). Comparing strategies for treating emotion perception deficits in traumatic brain injury. Journal of Head Trauma Rehabilitation, 23(2), 103-115.

Boyd, T.M., \& Sautter, S.W. (1993). Route-finding: A measure of everyday executive functioning in the head-injured adult. Applied Cognitive Psychology, 7, 171-181.

Braden, C., Hawley, L., Newman, J., Morey, C., Gerber, D., \& Harrison-Felix, C. (2010). Social communication skills group treatment: A feasibility study for persons with traumatic brain injury and comorbid conditions. Brain Injury, 24(11), 1298-1310.

Butter, C.M., \& Kirsch, N. (1992). Combined and separate effects of eye patching and visual stimulation on unilateral neglect following stroke. Archives of Physical Medicine and Rehabilitation, 73, 11331139.

Butter, C.M., \& Kirsch, N. (1995). Effect of lateralized kinetic visual cues on visual search in patients with unilateral spatial neglect. Journal of Clinical and Experimental Neurospsychology, 17(6), 856-867.

Carlson, L.A., Holscher, C., Shipley, T.F., \& Dalton, R.C. (2010). Getting lost in buildings. Current Directions in Psychological Science, 19, 284-289.

Channon, S., \& Crawford, S. (2010). Mentalising and social problem solving after brain injury. Neuropsychological Rehabilitation, 20(5), 739-759.

Chaytor, N., \& Schmitter-Edgecombe, M. (2003). The ecological validity of neuropsychological tests: a review of the literature on everyday cognitive skills. Neuropsychological Review, 13(4), 181-197.

Cho, Y.S., \& Sohlberg, M.M. (2014, February). Training adults with brain injury how to help-seek when lost: A pilot study. Poster presented at the meeting of the International Neuropsychological Society, Seattle, WA.

Cho, Y.S., Sohlberg, M.M., \& Boyd, T.M. (2012). Normative data and comparative performance across settings on a modified wayfinding task. Department of Special Education and Clinical Sciences: Communication Disorders and Sciences, University of Oregon, Eugene, Oregon.

Dahlberg, C.A., Cusick, C.P., Hawley, L.A., Newman, J.K., Morey, C.E., Harrison-Felix, C.L., \& Whiteneck, G.G. (2007). Treatment efficacy of social communication skills training after traumatic brain injury: A randomized treatment and deferred treatment controlled trial. Archives of Physical Medicine and Rehabilitation, 88, 1561-1573.

D’Zurilla, T.J., \& Goldfried, M.R. (1971). Problem solving and behavior modification. Journal of Abnormal Psychology, 78(1), 107-126.

D’Zurilla, T.J., \& Nezu, A.M. (1982). Social problem solving in adults. In P.C. Kendall (Ed.), Advances in cognitive-behavioral research and therapy ( $\mathrm{pp}$. 202-274). New York, NY: Academic Press.

D’Zurilla, T. J., \& Nezu, A.M. (1990). Development and preliminary evaluation of the Social Problem-Solving Inventory (SPSI). Psychological 
Assessment: A Journal of Consulting and Clinical Psychology, 2, 156-163.

D'Zurilla, T.J., \& Nezu, A.M. (2007). Problem-solving therapy: A positive approach to clinical intervention. New York, NY: Springer.

D'Zurilla, T.J., Nezu, A.M., \& Maydeu-Olivares, A. (2004). Social problem solving: Theory and assessment. In E.C. Chang, T.J. D'Zurilla \& L.J. Sanna (Eds.), Social problem solving: Theory, research and training (pp. 11-28). Washington, DC: American Psychological Association.

Engelmann, S., \& Carnine, D. (1982). Theory of instruction: Principles and applications. New York, NY: Iverington.

Evans, J.J. (2009). Rehabilitation of executive function: an overview. In M. Oddy, \& A. Worthington (Eds.), The rehabilitation of executive disorders. New York, NY: Oxford University Press.

Gourash, N. (1978). Help-seeking: A review of the literature. American Journal of Community Psychology, 6(5), 413-423.

Hawley, L.A., \& Newman, J.K. (2010). Group interactive structured treatment (GIST): A social competence intervention for individuals with brain injury. Brain Injury, 24(11), 1292-1297.

Heish, M.Y., Ponsford, J., Wong, D., Schonberger, M., McKay, A., \& Haines, K. (2013). Development of motivational interviewing programme as a prelude to CBT for anxiety following traumatic brain injury. Neuropsychological Rehabilitation: An International Journal, 22(4), 563-584.

Helffenstein, D.A., \& Wechsler, F.S. (1982). The use of interpersonal process recall (IPR) in the remediation of interpersonal and communication skill deficits in the newly brain-injured. Clinical Neuropsychology, 4(3), 139-142.

Horner, R.H., Sprague, J., \& Wilcox, B. (1982). Constructing general case programs for community activities. In B. Wilcox \& G.T. Bellamy (Eds.), Design of high school programs for severely handicapped students (pp. 61-98). Baltimore, MD: Paul H. Brookes Publishing.

Kato, Y., \& Takeuchi, Y. (2003). Individual differences in wayfinding strategies. Journal of Environmental Psychology, 23, 171-188.

Kizony, R., Demayo-Dayan, T., Sinoff, G., \& Josman, N. (2011). Validation of the executive function routefinding task (EFRT) in people with mild cognitive impairment. Occupation, Participation and Health, 31(1), S47-S52.

Lemoncello, R., Sohlberg, M.M., Fickas, S., \& Prideaux, J. (2010). When directions fail: Investigation of getting lost behavior with adults with acquired brain injury. Brain Injury, 24(3), 550-559.

Lui, A.L., Hile, H., Kautz, H., Borriello, G., Brown, P.A., Harniss, M., \& Johnson, K. (2008). Indoor wayfinding: Developing a functional interface for individuals with cognitive impairments. Disability and Rehabilitation: Assistive Technology, 3(1-2), 6981.
MacFadden, A., Elias, L., \& Saucier, D. (2003). Males and females scan maps similarly, but give directions differently. Brain and Cognition, 53, 297-300.

Malouff, J.M., Thorsteinsson, E.B., \& Schutte, N.S. (2007). The efficacy of problem solving therapy in reducing mental and physical health problems: A meta-analysis. Clinical Psychology Review, 27, 4657.

Manchester, D., Priestley, N., \& Jackson, H. (2004). The assessment of executive functions: Coming out of the office. Brain Injury, 18(11), 1067-1081.

McDonald, S., Tate., R., Togher, L., Bornhofen, C., Long, E., Gertler, P., \& Bowen, R. (2008). Social skills treatment for people with severe, chronic acquired brain injuries: A multicenter trial. Archives of Physical Medicine and Rehabilitation, 89(9), 1648-1659.

Rabin, L.A., Burton, L.A., \& Barr, W.B. (2007). Utilization rates of ecologically oriented instruments among clinical neuropsychologists. Clinical Neuropsychology, 21(5), 727-743.

Rath, J.F., Hradil, A.L., Litke, D.R., \& Diller, L. (2011). Clinical applications of problem-solving research in neuropsychological rehabilitation: Addressing the subjective experience of cognitive deficits in outpatients with acquired brain injury. Rehabilitation Psychology, 55(4), 320-328.

Rath, J.F., Simon, D., Langenbahn, D.M., Sherr, R.L., \& Diller, L. (2003). Group treatment of problem solving deficits in outpatients with traumatic brain injury: A randomized outcome study. Neuropsychological Rehabilitation, 13, 461-488.

Rietdijk, R., Simpson, G., Togher, L., Power, E., \& Gillett, L. (2013). An exploratory prospective study of the association between communication skills and employment outcomes after severe traumatic brain injury. Brain Injury, 27(7-8), 812-818.

Robertson, R.H., \& Knight, R.G. (2008). Evaluation of social problem solving after traumatic brain injury. Neuropsychological Rehabilitation, 18(2), 236-250.

Saucier, D.M., Green, S.M., Leason, J., MacFadden, A., Bell, S., \& Elias, L.J. (2002). Are sex differences in navigation caused by sexually dimorphic strategies or by differences in the ability to use the strategies? Behavioral Neuroscience, 3, 403-410.

Schmidt, J., Fleming, J., Ownsworth, T., \& Lannin, N.A. (2012). Video feedback on functional task performance improves self-awareness after traumatic brain injury: A randomized controlled trial. Neurorehabilitation and Neural Repair, 27(4), 316-324.

Sherr, R.L., Langenbahn, D.M., Simon, D., Rath, J.F., \& Diller, L. (2003). Problem-solving group protocol. Unpublished manual, Rusk Institute of Rehabilitation Medicine, NYU School of Medicine, New York.

Silverberg, N.D., Hallam, B.J., Rose, A., Underwood, H., Whitfield, K., Thornton, A.E., \& Whittal, M.L. (2013). Cognitive-behavioral prevention of postconcussion syndrome in at-risk patients: A pilot randomized controlled trial. Journal of Head Trauma Rehabilitation, 28(4), 313-322. 
Skeleton, R.W., Ross, S.P., Nerad, L., \& Livingstone, S.A. (2006). Human spatial navigation deficits after traumatic brain injury shown in the arena maze, a virtual Morris water maze. Brain Injury, 20(2), 189203.

Sloan, S., \& Ponsford, J. (2013). Managing cognitive problems following TBI. In J. Ponsford, S. Sloan \& P. Snow (Eds.), Traumatic brain injury: Rehabilitation for everyday adaptive living (pp. 99-132). New York, NY: Psychology Press.

Snow, P. (2013). Communication competence following TBI: Assessment and management. In J. Ponsford, S. Sloan \& P. Snow (Eds.), Traumatic brain injury: Rehabilitation for everyday adaptive living (pp. 133163). New York, NY: Psychology Press.

Sohlberg, M.M., Boyd, T.M., \& Cho, Y.S. (2012, February). Normative data and comparative performance across settings on a modified wayfinding task. Poster session presented at International Neuropsychological Society in Montreal, Quebec, Canada.

Sohlberg, M.M., Fickas, S., Hung, P.-F., \& Fortier, A. (2007). A comparison of four prompt modes for route finding for community travelers with severe cognitive impairments. Brain Injury, 21(5), 531538 .

Sohlberg, M.M., \& Turkstra, L.S. (2011). Optimizing cognitive rehabilitation: Effective instructional methods. New York, NY: Guilford Press.
Spiers, H.J., \& Maguire, E.A. (2008). The dynamic nature of cognition during wayfinding. Journal of Environmental Psychology, 28, 232-249.

Spikman, J.M., Deelman, B.G., \& van Zomeren, A.H. (2000). Executive functioning, attention and frontal lesions in patients with chronic CHI. Journal of Clinical and Experimental Neuropsychology, 22, 325338 .

Van Asselen, M., Kessels, R.P.C., Frijns, C.J.M., Kappelle, L.J., Neggers, S.F.W., \& Postma, A. (2005). Object-location memory: A lesion-behavior mapping study in stroke patients. Brain and Cognition, 71, 287-294.

Wang, Y., Chan, R.C.K., \& Shum, D.H.K. (2014). Executive function impairments after traumatic brain injury. In H.S. Levin, D.H.K. Shum \& R.C.K. Chan (Eds.), Understanding traumatic brain injury: Current research and future directions (pp. 99-115). New York, NY: Oxford.

Webber, L.S., \& Charlton, J.L. (2001). Wayfinding in older adults. Clinical Gerontologist, 23(1/2), 168172.

Ylvisaker, M. (2003). Context-sensitive cognitive rehabilitation after brain injury: Theory and practice. Brain Impairment, 4(1), 1-16.

Ylvisaker, M. (2006). Self-coaching: A context-sensitive, person-centered approach to social communication after traumatic brain injury. Brain Impairment, 7(3), 246-258. 